\title{
Avance en el proceso de la invasión biológica del peludo Chaetophractus villosus (Dasypodidae) en la Isla Grande de Tierra del Fuego: un nuevo desafío binacional
}

\author{
Advance in the biological invasion process of the \\ large hairy armadillo Chaetophractus villosus (Dasypodidae) in \\ Tierra del Fuego Island: a new binational challenge
}

José L. Cabello ${ }^{1, *}$, Alejandro E. J. Valenzuela ${ }^{2,3}$ \& Christopher B. Anderson ${ }^{3,4}$

\section{Resumen}

Las invasiones biológicas en islas por parte de mamíferos han sido identificadas como una de las principales causas de pérdida de la biodiversidad a nivel mundial, pero su investigación y manejo están sesgados hacia aquellas especies que se encuentran en la etapa final de la invasión. En este sentido, surge la necesidad de mantener actualizada la información sobre el proceso de una invasión biológica para que los tomadores de decisiones y gestores puedan implementar medidas de conservación que también abarquen el fenómeno en las fases de introducción y expansión. En particular, es importante evitar que en las etapas iniciales una invasión pase desapercibida, ya sea debido a características biológicas de la especie en sí, como tener hábitos crípticos o vivir en bajas densidades, pero también a factores sociales que puedan condicionar este sesgo, como en el caso de especies que se introducen en una jurisdicción política y se expanden a otra por no estar en la mirada de las autoridades y científicos de la nueva área invadida. Aquí presentamos antecedentes sobre la expansión del peludo (Chaetophractus villosus), una especie nativa en el sector continental de Chile y Argentina, pero exótica en la Isla Grande de Tierra del Fuego (TDF), que nos permiten alertar sobre el cambio a una fase de expansión en este proceso de invasión biológica. El peludo fue introducido en el sector argentino de TDF en 1982, donde ocupaba solo una franja a lo largo de la costa atlántica entre la ciudad de Río Grande y la Bahía San Sebastián y sus madrigueras estaban fuertemente asociadas con las cañerías enterradas de petróleo y gas. En 2014, se clasificó al peludo, en base a criterios de impacto ecológico, dentro de las especies prioritarias para el manejo. Sin embargo, a la fecha no existe ningún plan, proyecto ni legislación tendiente a controlar esta especie. Los nuevos registros de este trabajo amplían el área de distribución del peludo, tanto dentro del mismo sector argentino como hacia el sector chileno de TDF. Se detectó que las posibles causas y vectores de esta expansión incluyen tanto la dispersión natural como el transporte por humanos. Este caso demuestra nuevamente la falta de una visión binacional y socio-ecológica sobre el estudio y manejo de las invasiones biológicas, lo que conlleva consecuencias severas para la conservación de los sistemas ecológicos y socio-productivos. Se destaca que este proceso de invasión biológica binacional debe ser abordado a la brevedad y en conjunto por los gestores y tomadores de decisiones de ambos países.

1 Patagoniawildlife Ltda., Parcela $N^{\circ} 71$, Villa Andrea,

Punta Arenas, Chile. $\bowtie$ josecabello@patagoniawildlife.cl

2 Consejo Nacional de Investigaciones

Científicas y Técnicas (CONICET)

Av. Los Nires 2382, 9410 Ushuaia, Argentina

3 Instituto de Ciencias Polares, Ambiente y Recursos Naturales, Universidad Nacional de Tierra del Fuego, Av. Los Nires 2382, 9410 Ushuaia, Argentina

4 Centro Austral de Investigaciones Científicas (CADIC), Consejo Nacional de Investigaciones Científicas y Técnicas (CONICET), Dr. Bernardo Houssay 200, 9410

Ushuaia, Argentina 
Palabras clave: especies exóticas invasoras, invasiones biológicas, manejo de vida silvestre, Patagonia, Xenarthra.

\section{Abstract}

Biological invasions by mammals on islands have been identified as one of the main causes of global biodiversity loss, but research and management has been biased towards those species that are already in the final stage of the invasion. Therefore, it is necessary for managers and decision makers to keep updated on the biological invasion process of these species to implement conservation measures that also include introduction and expansion stages. In particular, it is important to prevent the initial invasion stages from going unnoticed, either due to the species' biological characteristics, such as cryptic habits or low densities, but also social factors that could condition this bias, such as species that were introduced in one political jurisdiction and expanded to another whose authorities and scientists were unaware of the newly invaded area. We present new records about the expansion of the large hairy armadillo (Chaetophractus villosus), a native species in the Chilean and Argentine mainland, but an exotic on Tierra del Fuego Island (TDF), that allow us to alert a change to the expansion stage in this biological invasion process. The armadillo was introduced in the Argentine side of TDF in 1982, where it occupied a thin strip of the Atlantic coast between Río Grande city and San Sebastián Bay, and their burrows were highly associated with natural gas and oil pipelines. In 2014, the armadillo was classified, based on its ecological impacts, among the priority species for management; however to date we found no plan, project or legislation focused on controlling this species' invasion. This work's new records extend the armadillo's distribution, on both the Argentine and Chilean sectors of TDF. We detected both natural dispersion and human transportation as possible causes and vectors of their expansion. This case again demonstrates the lack of a binational and socio-ecological perspective on the study and management of biological invasions, which has severe consequences for the conservation of native ecosystems. We highlight that this case has become a binational biological invasion process that should be quickly addressed together between the managers and decision makers of both countries.

Key words: biological invasions, invasive exotic species, Patagonia, wildlife management, Xenarthra.

Las invasiones biológicas son consideradas como una de las principales causas de pérdida de biodiversidad y del cambio ecológico global provocado por el ser humano (Vitousek et al. 1997). En particular, la introducción de mamíferos en sistemas insulares afecta las especies nativas, llevando a la extinción local o global de algunas de ellas (Courchamp et al. 2003). Para el Archipiélago Fueguino, Valenzuela et al. (2014) encontraron que incluso las especies exóticas invasoras han llegado a dominar el ensamble, con el $62,1 \%$ de los mamíferos presentes siendo introducidos. En este contexto, también es llamativo que dentro del Cono Sur de América, la ecorregión de los bosques subantárticos tenga el mayor número de mamíferos introducidos (Ballari et al. 2016), a pesar de ser considerado por ONGs internacionales como una de las últimas áreas "silvestres y prístinas" en el mundo (la palabra es wilderness en inglés, véase Mittermeier et al. 2003). Asimismo, en particular por la falta de información e investigación sobre los mamíferos exóticos invasores en el Archipiélago Fueguino (Valenzuela et al. 2014), en la Patagonia (Anderson \& Valenzuela, 2014), en la Argentina (Novillo \& Ojeda, 2008), en Chile (Jaksic, 1998; Jaksic et al. 2002) e incluso en el Cono Sur de Sudamérica (Ballari et al. 2016), tanto los académicos como los gestores y tomadores de decisiones han destacado la importancia de mantener actualizada la información respecto a las especies exóticas invasoras con el objetivo de poder utilizarla en la toma de decisiones (Sanguinetti et al. 2014; Valenzuela, 2014; Valenzuela et al. 2016), dado que la investigación prioriza la cuantificación de los impactos en la etapa de invasión y no se presta suficiente atención a las fases de introducción y expansión (Anderson \& Valenzuela, 2014).

Conocido localmente como peludo, quirquincho, piche o armadillo, Chaetophractus villosus (Desmarest, 1804) es una especie nativa de Patagonia continental, que constituye la más reciente introducción documentada de un vertebrado terrestre en el Archipiélago Fueguino 
(Poljak et al 2007, Valenzuela et al. 2014). Hacia 1975, esta especie expandió su límite sur hasta la costa del Estrecho de Magallanes (Atalah, 1975), que presupone una barrera geográfica natural (Poljak et al. 2007). El primer evento documentado de introducción de peludo en la Isla Grande de Tierra del Fuego (TDF) data de 1982 en la Estancia Cullen (Argentina), donde ocho individuos, probablemente traídos de la Provincia de Buenos Aires, fueron liberados por razones estéticas (Poljak et al. 2007; Ballari et al. 2016). Posteriormente, existieron otros eventos de introducción, como por ejemplo: en 2004-2005 cuando empleados de las compañías petroleras reportaron haber traído individuos desde la Provincia de Santa Cruz para alimentación (Poljak et al. 2007). Estos autores describen que hasta 2007, la especie ocupaba solo una franja a lo largo de la costa atlántica entre las Sierras Cabeza del León hasta el Cabo Domingo, cubriendo aproximadamente $484 \mathrm{~km}^{2}$, y sus madrigueras estaban fuertemente asociadas con las cañerías enterradas de petróleo y gas debido a que para su instalación se remueve el suelo y adicionalmente podrían generar un refugio térmico durante el invierno (Poljak et al. 2007). A su vez, en ese momento, se presumía que el Río Grande actuaría como una barrera geográfica para la expansión geográfica de la especie y hasta esa fecha no existían reportes ni registros confirmados sobre su presencia en el sector chileno al oeste de TDF.

Si bien podrían ser útiles más investigaciones específicas respecto al impacto de esta especie en TDF, ya se sabe que las actividades de excavación del peludo afectan la estructura del hábitat (Valenzuela et al. 2014), facilitando la erosión, generando suelo desnudo, mezclando los horizontes del suelo y modificando sus propiedades (Abba et al. 2007). Además, pueden causar daño a casas, construcciones y a la infraestructura asociada a la industria del petróleo y el transporte de gas, principalmente las cañerías subterráneas (Long, 2003; Poljak et al. 2007; Ballari et al. 2016). Dado que $C$. villosus se alimenta de plantas, frutos, semillas, insectos, huevos y pichones de aves que anidan en el suelo, e incluso pequeños vertebrados como ratones (Long, 2003; Poljak et al. 2007), sería inevitable que su expansión afecte la biodiversidad y tramas tróficas de la isla.
En el caso de TDF, además del impacto en la infraestructura humana, la presencia de peludos podría afectar la reproducción del Canquén Colorado (Chloephaga rubidiceps, Sclater 1861; Ballari et al. 2016), especie amenazada de extinción y cuyas poblaciones están declinando (Cossa et al. 2017), y competir con el tuco-tuco magallánico (Ctenomys magellanicus, Bennet 1836), roedor nativo en estado de conservación vulnerable que ocupa la misma área que el peludo y que también excava el suelo para generar madrigueras (Fasanella et al. 2013). Asimismo, el peludo es conocido por ser posible vector de diversas enfermedades humanas, como el mal de Chagas y la triquinosis (Long, 2003). Ya en 2014, y pese a que el peludo todavía se encontraba en una etapa relativamente temprana del proceso de invasión, surgió como una especie prioritaria a ser manejada entre todo el ensamble de vertebrados exóticos de TDF, según criterios de impacto ecológico (Valenzuela et al. 2014), pero en la actualidad, no existen medidas formales de manejo o control para esta especie en la región. Diversas posibilidades podrían explicar esta situación: i) continúan primando las motivaciones que originaron su introducción (valores estéticos y de alimentación); ii) como la especie fue introducida hace relativamente poco tiempo y su distribución se mantuvo limitada hasta recientemente, la percepción del problema no ha llegado a influir en la agenda pública todavía, dado que tanto los investigadores como los gestores se enfocaron más en otras especies con impactos evidentes desde hace muchos años, como el castor (Castor canadensis, Kuhl 1820) y el visón americano (Neovison vison, Schreber 1777) (Anderson \& Valenzuela, 2014); iii) por tratarse de una especie nativa del continente (de donde son originarios la mayoría de los pobladores de TDF), no es percibida como una especie exótica invasora, ya que la mayoría de los residentes de la parte argentina de TDF desconocen los mamíferos nativos a la isla (Anderson et al. 2016); y iv) del mismo modo, por ser una especie nativa en ambos países (Argentina y Chile), existe un vacío legal respecto a su situación como introducida, lo cual complica cualquier medida de manejo dado que es una especie protegida por ley.

En este contexto, se confirma la necesidad de mantener actualizada la información sobre el 


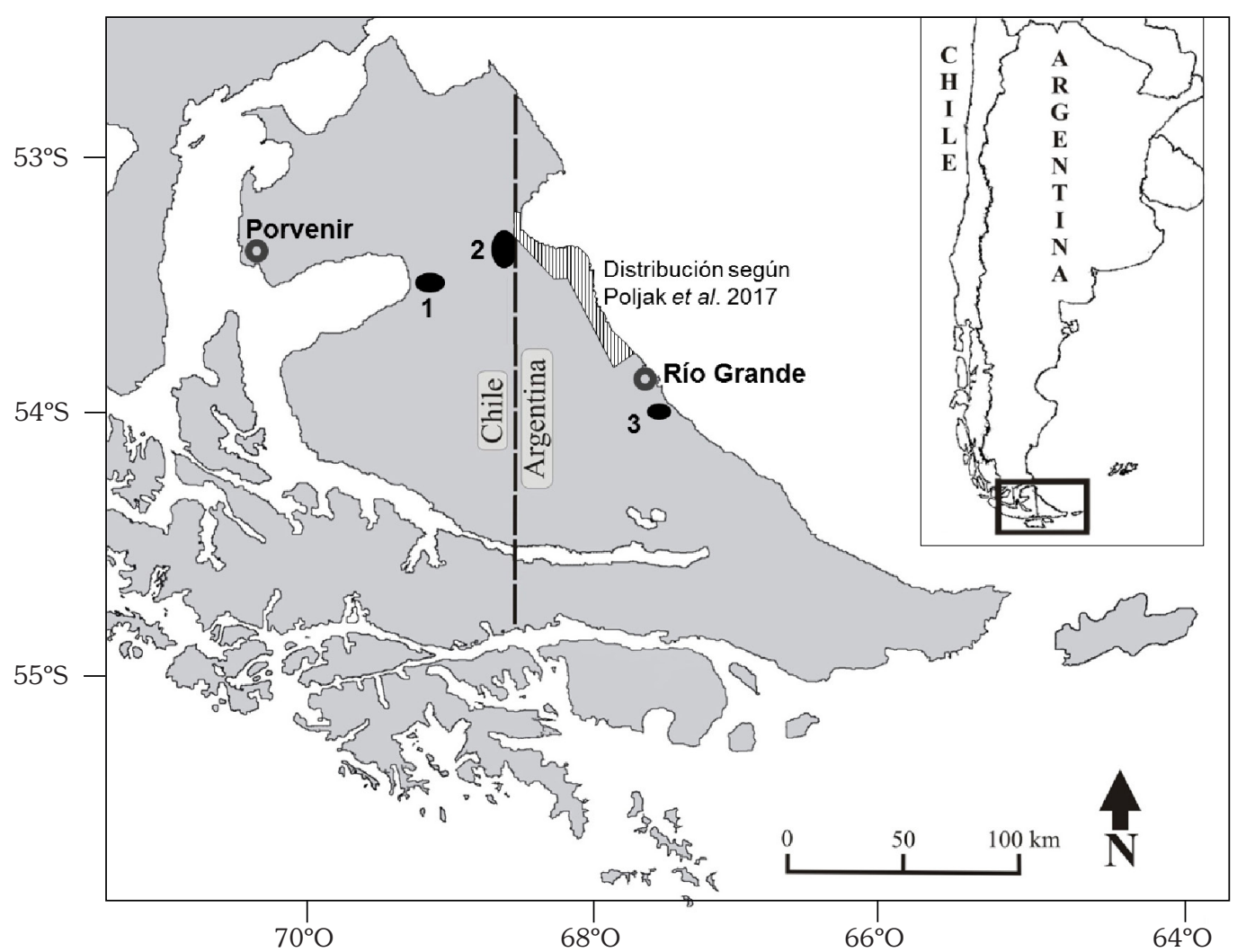

Fig. 1. Mapa de la Isla Grande de Tierra del Fuego donde se muestra la distribución del peludo (Chaetophractus villosus) como especie exótica incluyendo la distribución descripta por Poljak et al. (2007) en líneas verticales y los nuevos registros descriptos en este trabajo en negro.

Donde 1: Cuenca del Río Marazzi; 2: San Sebastián; y 3: Estancia San Luis.

proceso de las invasiones biológicas, desde la etapa de introducción hasta la invasión propiamente dicha, para que los gestores y tomadores de decisiones puedan desarrollar respuestas acordes a la fase en que se encuentra la especie y evitar los efectos más nocivos de la invasión en sí (Sanguinetti et al. 2014) y en particular para especies poco visibles, carismáticas y de relativa baja densidad, como puede ser el caso del peludo. En el presente artículo, reportamos nuevos registros que amplían el área de distribución, lo cual indica que el peludo está en una etapa de expansión tanto en el sector argentino como en el chileno de TDF (Fig. 1). A partir de un diagnóstico de la situación actual de este proceso de invasión biológica también detectamos necesidades conceptuales y prácticas que se tienen que tener en cuenta para manejar estos problemas como sistemas socio-ecológicos.

En el sector argentino, el encargado de la Estancia San Luis ha informado diversas observaciones de peludos en su establecimiento (Ivon Roberts, com. pers.), que se encuentra al sur del Río Grande. Esta expansión pudo estar ocasionada por una dispersión natural de la especie o por nuevas translocaciones por parte de humanos. Más allá del vector, queda demostrada la capacidad de la especie de superar lo que podría haber constituido una barrera natural, principalmente por el ancho y caudal del Río Grande, y de este modo expandirse hacia el sur, pudiendo llegar incluso a la zona del bosque subantártico (Fig. 1).

En el sector chileno de TDF, detectamos diversos registros que permiten demostrar su presencia en dicho país y que suman así un nuevo mamífero exótico al listado actual de Chile (ver Jaksic et al. 1998; 2002; Anderson et al. 2006). El primer dato que confirmó la presencia del peludo en Chile fue a partir de una piel de un individuo 
que fue cazado cerca de San Sebastián chileno (una zona limítrofe con Argentina) en el año 2004 (Juan Ferrada, Funcionario SAG Porvenir, com. pers., Fig. 2A). El segundo constituye un avistamiento directo realizado por uno de los autores (J.L. Cabello) en el año 2007, también en el sector chileno de San Sebastián, 53¹9'8.17'S $68^{\circ} 42$ '33.37"O; mientras que el tercero corresponde a un avistamiento con fotografía (Fig. 2B), de dos peludos copulando en la misma área, 5319'4.35"S 6851'57.39”O, registrado por un investigador chileno también en 2007 (Claudio Moraga, com. pers.). Adicionalmente, existe una serie de registros en la cuenca del Río Marazzi en las cercanías a Bahía Inútil, 53²8'12.13"S 69 $16^{\prime} 45.48^{\prime \prime} O$, donde personal de las estancias Tres Hermanos y Tres Arroyos han comunicado varios avistamientos entre los años 2009 y 2015 (Juan Robertson Stipicic, com. pers.). Asimismo, arqueólogos de la Universidad de Magallanes (UMAG) reportaron haber encontrado madrigueras de peludos durante excavaciones arqueológicas en la misma zona en el año 2014 (Flavia Morello Repetto, com. pers.).

Respecto a las vías y los vectores de esta expansión, los registros correspondientes a la zona chilena de San Sebastián, que es aledaña al área originalmente invadida, probablemente correspondan a una dispersión natural de la especie. Además, dicho sector presenta características similares al ya ocupado del lado argentino, con extensas redes subterráneas de tuberías de petróleo, lo que beneficiaría el establecimiento de madrigueras, según Poljak et al. (2007). Sin embargo, la información resultante de consultas informales con los habitantes del área de la ciudad de Porvenir y la Bahía Gente Grande sugiere que existe otro vector de expansión responsable de la presencia del peludo en esa zona, debido al salto en la distribución hasta la costa oeste de la isla sin registros intermedios. Los lugareños consultados indicaron que el transporte y la liberación por humanos continúa siendo el factor más importante para explicar su presencia. Reportaron que los camioneros que transportan carga desde el continente hacia TDF suelen recoger estos animales por curiosidad o como fuente de alimento en el área continental chilena y transportarlos a la isla, donde a veces son liberados, en particular antes de pasar por el control fronterizo chileno-argentino ubicado en la localidad de San Sebastián.

Este trabajo amplia la distribución del peludo como especie exótica en TDF, incluyendo los primeros registros formales para Chile y también

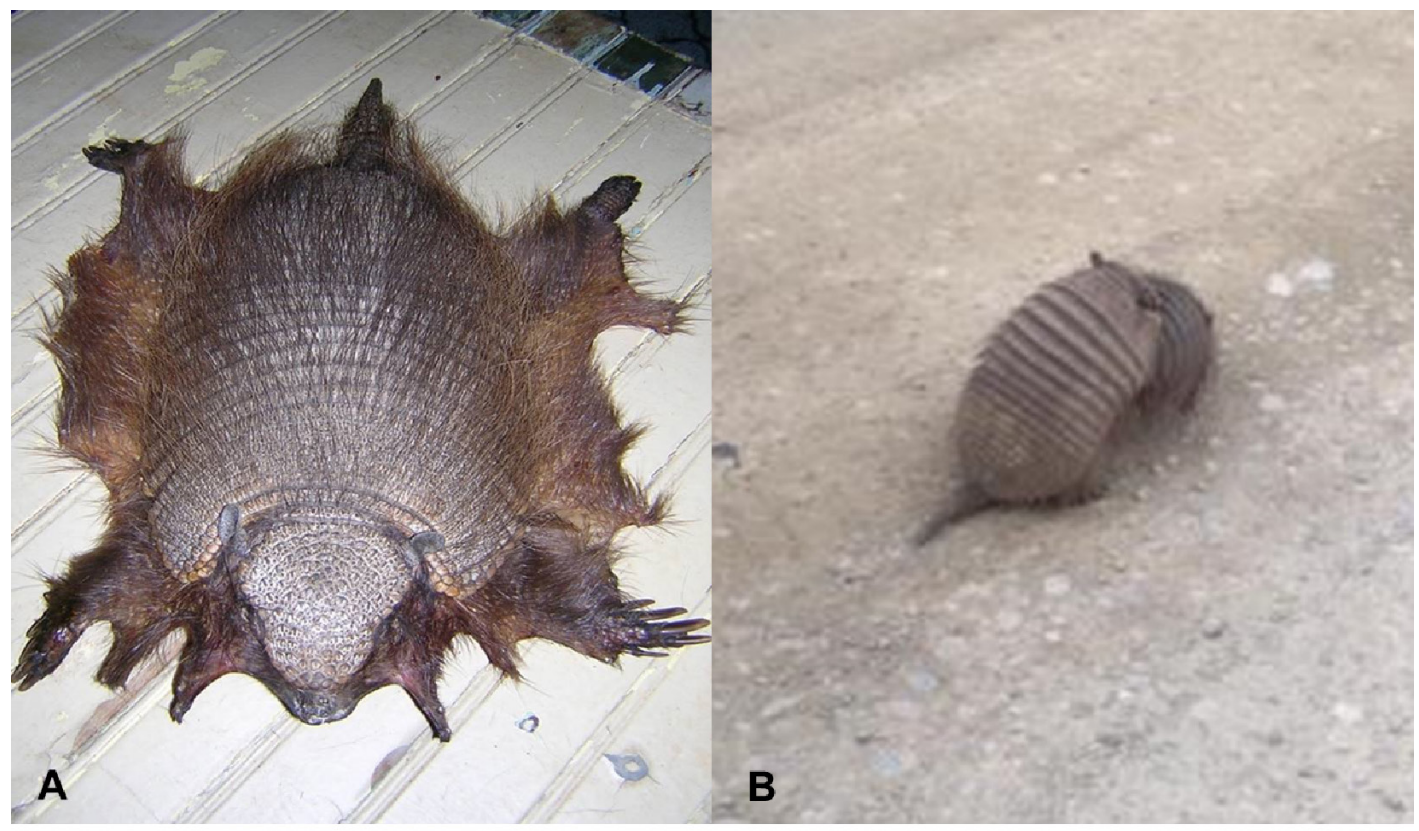

Fig. 2. Registros de peludo (Chaetophractus villosus) en el sector chileno de San Sebastián. A: piel de individuo cazado (Foto: J.L. Cabello); B: individuos copulando en el área (Foto: V. Valenzuela). 
su expansión en el lado argentino de la isla a otro bioma - desde la estepa patagónica hacía el ecotono entre la estepa y el bosque subantártico. También, se vuelve a repetir el fenómeno que una especie llega a ser una problemática binacional, visto además con el castor norteamericano, el visón americano y el salmón Chinook (Oncorhynchus tshawytscha, Walbaum 1792; Valenzuela et al. 2014), siendo no percibida por las autoridades e investigadores del vecino país hasta ya haberse establecido, expandido y llegado a ser "invasora". Considerando que en la actualidad la superficie invadida es relativamente poca (y probablemente también el número de animales), aún existe una ventana de oportunidad para que los gestores y tomadores de decisiones de ambos países tomen las medidas conjuntas necesarias para controlar este proceso de invasión biológica, mientras que sea posible. Asimismo, se evidencia nuevamente la necesidad de cambiar la forma de concebir las invasiones biológicas que en lugar de un fenómeno meramente ecológico en realidad constituyen problemáticas socio-ecológicas. En este sentido, además de entender la dinámica poblacional o el impacto ecosistémico de la especie se requiere un esfuerzo similar para estudiar y mejorar la legislación y el manejo sobre los aspectos sociales, como los sistemas de transporte tanto en los pasos fronterizos como en el ingreso a TDF. Y por otro lado generar una fuerte campaña de construcción de sentido de lugar y pertinencia de los residentes de TDF para que conozcan las especies nativas $y$ sean capaces de reconocer las consecuencias del transporte de fauna silvestre, dirigida especialmente a conductores, para tratar de prevenir nuevas introducciones.

\section{AGRADECIMIENTOS}

El presente trabajo constituye parte de la tesis de Magister de JLC en la UMAG. Agradecemos a las personas que gentilmente nos brindaron la información sobre estos registros.

\section{LITERATURA CITADA}

Abba, A. M., Vizcaíno, S. F., \& Cassini, M. G. (2007). Effects of land use on the distribution of three species of armadillos in the Argentinean Pampas. Journal of Mammology, 88, 502-507.

Anderson, C. B., Rozzi, R., Torres-Mura, J. C., Mcgehee, S. M., Sherriffs, M. F., Schüttler, E., \& Rosemond, A. D. (2006). Exotic vertebrate fauna in the remote and pristine sub-Antarctic Cape Horn Archipelago, Chile. Biodiversity and Conservation, 15, 3295-3313.

Anderson, C. B., \& Valenzuela, A. E. J. (2014). Do what I say, not what I do. Are we linking research and decision-making about invasive species in Patagonia? Ecología Austral, 24, 193-202.

Anderson, C. B., Valenzuela, A. E. J., van Aert, P., Malizia, M., Car, V., \& Ader, N. (2016). Informe final. Proyecto PEININ "Evaluación socio-ecológica del Parque Nacional de Tierra del Fuego". Universidad Nacional de Tierra del Fuego y Administración de Parques Nacionales.

Atalah, A. (1975). Presencia de Chaetophractus villosus (Edentata Dasypodidae) nueva especie para la Región de Magallanes, Chile. Anales del Instituto de la Patagonia, 6, 169-171.

Ballari, S., Anderson, C. B., \& Valenzuela, A. E. J. (2016). Understanding trends in biological invasions by introduced mammals in the southern South America: a review of research and management. Mammal Reviews, 46, 229-240.

Cossa, N. A., Fasola, L., Roesler, I., \& Reboreda, J. C. (2017). Ruddy-headed Goose Chloephaga rubidiceps: former plague and present protected species on the edge of extinction. Bird Conservation International, 27, 269-281.

Courchamp, F., Chapuis, J. L., \& Pascal, M. (2003). Mammal invaders on islands: impact, control and control impact. Biological Reviews, 78, 347-383.

Fasanella, M., Bruno, C., Cardoso, Y., \& Lizarralde, M. (2013). Historical demography and spatial genetic structure of the subterranean rodent Ctenomys magellanicus in Tierra del Fuego (Argentina). Zoological Journal of the Linnean Society, 169, 697-710.

Jaksic, F. M., Iriarte, J. A., Jiménez, J. E., \& 
Martínez, D. R. (2002). Invaders without frontiers: crossborder invasions of exotic mammals. Biological invasions, 4, 157173.

Jaksic, F. M. (1998). Vertebrate invaders and their ecological impacts in Chile. Biodiversity \& Conservation, 7, 1427-1445.

Long, J. L. (2003). Introduced Mammals of the World: Their History, Distribution and Influence. CSIRO Publishers, Collingwood. Mittermeier, R. A., Mittermeier, C. G., Brooks, T. M., Pilgrim, J. D., Konstant, W. R., da Fonseca, G. A. B., \& Kormos, C. (2003). Wilderness and biodiversity conservation. Proceedings of the National Academy of Sciences of the United States of America, 100(18),10309-10313.

Novillo, A., \& Ojeda, R. A. (2008). The exotic mammals of Argentina. Biological Invasions, 886,1333-1344.

Poljak, S., Escobar, J., Deferrari, G., \& Lizarralde, M. (2007). Un nuevo mamífero en la Tierra del Fuego: el "peludo" Chaetophractus villosus (Mammalia, Dasypodidae) en Isla Grande. Revista Chilena de Historia Natural, 80, 285-294.

Sanguinetti, J., Buria, L., Malmierca, L., Valenzuela, A. E. J., Núñez, C., Pastore, H., Chauchard, L.,...Chehébar, C. (2014).
Manejo de especies exóticas invasoras en Patagonia, Argentina: Priorización, logros y desafíos de integración entre ciencia y gestión identificados desde la Administración de Parques Nacionales. Ecología Austral, 24, 183-192. Valenzuela, A. E. J. (2014). Integrando la Investigación y la Gestión de Especies Exóticas Invasoras en la Patagonia. Ecología Austral, 24,133-134.

Valenzuela, A. E. J., Anderson, C. B., Fasola, L., \& Cabello, J. L. (2014). Linking invasive exotic vertebrates and their ecosystem impacts in Tierra del Fuego to test theory and determine action. Acta Oecologica, 54, 110-118.

Valenzuela, A. E. J., Sepúlveda, M., Cabello, J. L., \& Anderson, C. B. (2016). El visón americano en Patagonia: una revisión binacional sobre las perspectivas de investigación, históricas, socio-ecológicas y de manejo de este carnívoro exótico invasor. Mastozoología Neotropical, 23, 289-304.

Vitousek, P. M., D’Antonio, C. M., Loope, L. L., Rejmanek, M., \& Westbrooks, R. (1997). Introduced species: a significant component of human-caused global change. New Zealand Journal of Ecology, 21, 1-16. 
